Discrete Comput Geom 27:387-393 (2002)

DOI: $10.1007 / \mathrm{s} 00454-001-0076-1$

\title{
Helly-Type Theorems on the Homology of the Space of Transversals
}

\author{
J. Bracho and L. Montejano
}

Instituto de Matemáticas, UNAM,

Circuito exterior, Ciudad Universitaria,

C.P. 04510, México DF, Mexico

\begin{abstract}
In this paper we "measure" the size of the set of $n$-transversals of a family $F$ of convex sets in $R^{n+k}$ according to its homological complexity inside the corresponding Grassmannian manifold. Our main result states that the "measure" $\mu$ of the set of $n$-transversals of $F$ is greater than or equal to $k$ if and only if every $k+1$ members of $F$ have a common point and also if and only if for some integer $m, 1 \leq m \leq n$, and every subfamily $F^{\prime}$ of $F$ with $k+2$ members, the "measure" $\mu$ of the set of $m$-transversals of $F^{\prime}$ is greater than or equal to $k$.
\end{abstract}

\section{Introduction}

For a family $F=\left\{A^{1}, \ldots, A^{d}\right\}$ of $d$ convex sets in $R^{n+k}$, let $T_{n}(F)$ be the set of $n$ transversals to $F$, that is, the set of all $n$-planes in $R^{n+k}$ which intersect every member of $F$.

If $X$ is a set of $n$-planes in $R^{n+k}$, we say that $\mu(X) \geq r$ if $X$ has "homologically" as many $n$-planes as the set of $n$-planes through the origin in $R^{n+r}$. Thus, $\mu$ "measures" the homological complexity of $X$ inside the corresponding Grassmannian manifold. We use this "measure" to prove that if subfamilies of $F$ with few members have enough transversals of small dimension, then the whole family $F$ has many transversals of a fixed dimension. That is, after a formal definition of $\mu$, in Section 2, we prove in Section 3 the equivalence of the following three properties:

- Every $k+1$ members of $F$ have a point in common.

- $\mu\left(T_{n}(F)\right) \geq k$.

- For some integer $m$ where $1 \leq m \leq n$ and every subfamily $F^{\prime}$ of $F$ with $k+2$ members, $\mu\left(T_{m}\left(F^{\prime}\right)\right) \geq k$. 
The first equivalence can be thought of as a homological version of Horn and Klee's classical results [5], [6]. See also [4]. They proved that the following assertions are equivalent:

(a) Every $k+1$ members of $F$ have a point in common.

(b) Every linear $n$-subspace of $R^{n+k}$ admits a translate which is a member of $T_{n}(F)$.

(c) Every $(n-1)$-plane $\Lambda$ lies in a member of $T_{n}(F)$.

First note that (b) is just assertion (c), when $\Lambda$ lies at infinity. In fact, the set of all $n$-planes that contain $\Lambda$ is a manifold embedded in the corresponding Grassmannian manifold, which represents an element of its cohomology. So, by using the product structure of the cohomology we shall prove that

$$
\mu\left(T_{n}(F)\right) \geq k \quad \Rightarrow \quad(b) \text { and }(c) .
$$

If $X$ is a set of $n$-planes in $R^{n+k}$ and for every linear $n$-subspace of $R^{n+k}$ we can choose a translate which is a member of $X$, then $\mu\left(T_{n}(F)\right)$ is not necessarily greater than or equal to $k$, unless, of course, according to our definition of $\mu$, the choice can be done continuously. If $X=T_{n}(F)$, the existence of a member of $T_{n}(F)$ parallel to every linear $n$-subspace of $R^{n+k}$ implies that we can choose this member continuously and hence that

$$
\mu\left(T_{n}(F)\right) \geq k \quad \Leftrightarrow \quad(b) \text { and }(c) .
$$

The spirit of the complete equivalences follows the topological study of the space of transversals initiated in [1] and [2].

We consider Euclidean $n$-space $R^{n}$ and complete it to the $n$-projective space $P^{n}$ by adding the hyperplane at infinity. Let $G(n+k, n)$ be the Grassmannian $n k$-manifold of all $n$-planes through the origin in Euclidean space $R^{n+k}$. Although we summarize what we need in Section 2, good references for the homology and cohomology of Grassmannian manifolds are [7], [9] and [3]; see also [8]. In this paper we use reduced Cech-homology and cohomology with $Z_{2}$-coefficients.

\section{The Topology of Grassmannian Manifolds}

Let $\lambda_{1}, \ldots, \lambda_{n}$ be a sequence of integers such that $0 \leq \lambda_{1} \leq \cdots \leq \lambda_{n} \leq k$. We denote by:

(2.1) $\left\{\lambda_{1}, \ldots, \lambda_{n}\right\}=\left\{H \in G(n+k, n) \mid \operatorname{dim}\left(H \cap R^{\lambda_{j}+j}\right) \geq j, j=1, \ldots, n\right\}$. For example, $\{0, \lambda, \ldots, \lambda\}=\left\{H \in G(n+k, n) \mid R^{1} \subset H \subset R^{n+\lambda}\right\}$ and $\{k-\lambda, \ldots, k-$ $\lambda, k\}=\left\{H \in G(n+k, n) \mid \operatorname{dim}\left(H \cap R^{n-1+k-\lambda}\right) \geq n-1\right\}$.

(2.2) It is known that $\left\{\lambda_{1}, \ldots, \lambda_{n}\right\} \subset G(n+k, n)$ is a closed connected $\lambda$-manifold, where $\lambda=\sum_{1}^{n} \lambda_{i}$, except possibly for a closed connected subset of codimension three. Thus, $H^{\lambda}\left(\left\{\lambda_{1}, \ldots, \lambda_{n}\right\} ; Z_{2}\right)=Z_{2}=H_{\lambda}\left(\left\{\lambda_{1}, \ldots, \lambda_{n}\right\} ; Z_{2}\right)$. Let $\left(\lambda_{1}, \ldots, \lambda_{n}\right) \in$ $H_{\lambda}\left(G(n+k, n) ; Z_{2}\right)$ be the $\lambda$-cycle which is induced by the inclusion $\left\{\lambda_{1}, \ldots, \lambda_{n}\right\} \subset$ $G(n+k, n)$. These cycles are called Schubert-cycles. A canonical basis for $H_{\lambda}(G(n+$ 
$\left.k, n) ; Z_{2}\right)$ consists of all Schubert-cycles $\left(\xi_{1}, \ldots, \xi_{n}\right)$ such that $0 \leq \xi_{1} \leq \cdots \leq \xi_{n} \leq k$ and $\sum_{1}^{n} \xi_{i}=\lambda$

(2.3) We denote by $\left[\lambda_{1}, \ldots, \lambda_{n}\right] \in H^{\lambda}\left(G(n+k, n) ; Z_{2}\right)$ the $\lambda$-cocycle whose value is one for $\left(\lambda_{1}, \ldots, \lambda_{n}\right)$ and zero for any other Schubert-cycle of dimension $\lambda$. Thus a canonical basis for $H^{\lambda}\left(G(n+k, n) ; Z_{2}\right)$ consists of all Schubert-cocycles $\left[\xi_{1}, \ldots, \xi_{n}\right]$ such that $0 \leq \xi_{1} \leq \cdots \leq \xi_{n} \leq k$ and $\sum_{1}^{n} \xi_{i}=\lambda$.

The isomorphism $D: H_{\lambda}\left(G(n+k, n) ; Z_{2}\right) \rightarrow H^{n k-\lambda}\left(G(n+k, n) ; Z_{2}\right)$ given by $D\left(\left(\lambda_{1}, \ldots, \lambda_{n}\right)\right)=\left[k-\lambda_{n}, \ldots, k-\lambda_{1}\right]$ is the classical Poincaré Duality Isomorphism.

(2.4) By the above, if $X \subset G(n+k, n)$ is such that $X \cap\left\{\lambda_{1}, \ldots, \lambda_{n}\right\}=\emptyset$ and $i_{X}: X \rightarrow G(n+k, n)$ is the inclusion, then

$$
i_{X}^{*}\left(D\left(\left(\lambda_{1}, \ldots, \lambda_{n}\right)\right)\right)=i_{X}^{*}\left(\left[k-\lambda_{n}, \ldots, k-\lambda_{1}\right]\right)=0 .
$$

(2.5) Let $M(n+k, n)$ be the set of all $n$-planes in $R^{n+k}$. Thus, $G(n+k, n) \subset M(n+$ $k, n)$. We regard $M(n+k, n)$ as an open subset of $G(n+k+1, n+1)$, making the following identifications:

Let $z_{0} \in R^{n+k+1}-R^{n+k}$ be a fixed point and, without loss of generality, let $G(n+$ $k+1, n+1)$ be the space of all $(n+1)$-planes in $R^{n+k+1}$ through $z_{0}$. We identify $H \in M(n+k, n)$ with the unique $(n+1)$-plane $H^{\prime} \in G(n+k+1, n+1)$ which contains $H$ and passes through $z_{0}$. Thus

$$
G(n+k, n) \subset M(n+k, n) \subset G(n+k+1, n+1),
$$

where $M(n+k, n)$ is an open subset of $G(n+k+1, n+1)$ and $G(n+k, n) \subset$ $G(n+k+1, n+1)$ may be regarded as $\{0, k, \ldots, k\}$, the set of all $(n+1)$-planes in $R^{n+k+1}$ which contains $R^{1}$. In other words, if $j: G(n+k, n) \rightarrow G(n+k+1, n+1)$ is the inclusion, then $j\left(\left\{\lambda_{1}, \ldots, \lambda_{n}\right\}\right)=\left\{0, \lambda_{1}, \ldots, \lambda_{n}\right\}$. So, if $0 \leq \lambda \leq k$, then $\{0, \lambda, \ldots, \lambda\}$ as a subset of $M(n+k, n)$ is the set of all $n$-planes $H$ through the origin in $R^{n+k}$ with the property that $H \subset R^{n+\lambda}$.

If $X \subset M(n+k, n)$, then $i_{X}: X \rightarrow G(n+k+1, n+1)$ denotes the inclusion.

(2.6) Let $A$ be a subset of $X$, let $i: A \rightarrow X$ be the inclusion and let $\gamma \in H^{*}\left(X ; Z_{2}\right)$. We say that $\gamma$ is zero or not zero in $A$, provided $i^{*}(\gamma)$ is zero or not zero respectively, in $H^{*}\left(A ; Z_{2}\right)$.

Now we are ready to state our main definition which captures the basic idea of having as many $n$-planes as the set of all $n$-planes through the origin in $R^{n+r}$.

Definition. Let $X \subset M(n+k, n) \subset G(n+k+1, n+1)$. For $0 \leq r \leq k$, we say that the "measure" of $X$ is at least $r$,

$$
\mu(X) \geq r,
$$

if $[0, r, \ldots, r]$ is not zero in $X$.

It is easy to verify that if $\mu(X) \geq r$, then, for any integer $0 \leq r_{0} \leq r, \mu(X) \geq r_{0}$. Furthermore, observe that if $m>0$, then $X$ is also naturally contained in $M(n+m+k, n)$ and the definition of the "measure" $\mu$ is independent of $m$. 
Example 2.1. Let $F=\left\{A^{0}, \ldots, A^{d}\right\}$ be a family of convex sets. We say that $F$ has a cycle of transversal lines if there is a transversal line that moves continuously until it comes back to itself with the opposite orientation. Observe that $F$ has a cycle of transversal lines if and only if $\mu\left(T_{1}(F)\right) \geq 1$.

The following lemma will be very useful for our purposes.

Lemma 2.1. Let $X \subset M(n+k, n)$ be a collection of $n$-planes and let $H$ be an $r$-plane of $R^{n+k}, 1 \leq r \leq k$. If $\mu(X) \geq r$, then there is $\Gamma \in X$ such that $\pi_{H}(\Gamma)$ is a single point, where $\pi_{H}: R^{n+k} \rightarrow H$ is the orthogonal projection.

Proof. Let $Y \subset M(n+k, n)$ be the set of all $n$-planes $\Gamma$ in $R^{n+k}$ such that $\pi_{H}(\Gamma)$ is a single point. As in (2.5), we regard $Y \subset M(n+k, n)$ as a subset of $G(n+k+1, n+1)$. Let $\Delta$ be the $(n+k-r)$-plane in $R^{n+k+1}$ through $z_{0}$ orthogonal to the $(r+1)$-plane that contains $H$ and passes through $z_{0}$. Note that $\Gamma \in Y$ if and only if the $(n+1)$-plane $\Gamma^{\prime}$ that contains $\Gamma$ and passes through $z_{0}$ is such that $\operatorname{dim}\left(\Gamma^{\prime} \cap \Delta\right) \geq n$. Consequently, if we regard $Y$ as a subset of $G(n+k+1, n+1)$, by (2.1) and (2.5), $Y=\{k-r, \ldots, k-r, k\}$.

We regard $X$ as a subset of $G(n+k+1, n+1)$ and suppose that $X \cap Y=\emptyset$. Then, by $(2.4), i_{X}^{*}([0, r, \ldots, r])=0$, which means that $[0, r, \ldots, r]$ is zero in $X$, but this is a contradiction because $\mu(X) \geq r$. Then $X \cap Y \neq \emptyset$. This completes the proof of Lemma 2.1.

Remark 2.1. If, in the above proof, $k=r$ and $Y \subset M(n+k, n)$ is the set of all $n$-planes $\Gamma$ in $R^{n+k}$ such that $\Gamma \subset \Lambda$, where $\Lambda$ is an $(n-1)$-plane in $P^{n+k}$, then we obtain the following result. Let $X \subset M(n+k, n)$ be a collection of $n$-planes with the property that $\mu(X) \geq k$, then every linear $n$-subspace of $R^{n+k}$ admits a translate which is a member of $X$; and every $(n-1)$-plane $\Lambda$ lies in a member of $X$.

\section{The Space of Transversals}

Let $F=\left\{A^{0}, \ldots, A^{d}\right\}$ be a family of convex sets in $R^{n+k}$ and let $T_{n}(F)$, the space of $n$-transversals of $F$, be the subset of the Grassmannian manifold $M(n+k, n)$ of $n$-planes that intersect all members of $F$.

Before stating our first result we need the following technical lemma.

Lemma 3.1. Let $A^{0}, A^{1}, \ldots, A^{k}$ be $k+1$ convex sets in $R^{n+k}, n \geq 0$, such that $\bigcap_{0}^{k} A^{i}=\emptyset$. Then there is a $k$-dimensional linear subspace $H$ of $R^{n+k}$ with the property that $\bigcap_{0}^{k} \pi_{H}\left(A^{i}\right)=\emptyset$, where $\pi_{H}: R^{n+k} \rightarrow H$ is the orthogonal projection.

Proof. The proof is by induction on $k$. If $k=1$, the proof follows by the separation theorem for disjoint convex sets. Suppose the theorem is true for $k$, we will prove it for $k+1$.

Let $A^{0}, A^{1}, \ldots, A^{k+1}$ be $k+2$ convex sets in $R^{n+k}$, such that $\bigcap_{0}^{k+1} A^{i}=\emptyset$. Since $\left(\bigcap_{0}^{k} A^{i}\right) \cap A^{k+1}=\emptyset$, then there is a hyperplane $\Lambda$ that separates $\bigcap_{0}^{k} A^{i}$ from $A^{k+1}$. 
Suppose $\bigcap_{0}^{k} A^{i} \subset \Lambda^{-}$and $A^{k+1} \subset \Lambda^{+}$, where $\Lambda^{+}$and $\Lambda^{-}$are the closed half-spaces determined by $\Lambda$. Note that $\bigcap_{0}^{k}\left(A^{i} \cap \Lambda^{+}\right)=\emptyset$.

By the induction hypothesis, there is a $k$-dimensional linear subspace $H_{0}$ such that $\bigcap_{0}^{k} \pi_{H_{0}}\left(A^{i} \cap \Lambda^{+}\right)=\emptyset$. Let $H$ be a $(k+1)$-dimensional linear subspace containing $H_{0}$ and the one-dimensional linear subspace orthogonal to $\Lambda$. We shall prove that

$$
\bigcap_{0}^{k+1} \pi_{H}\left(A^{i}\right)=\emptyset .
$$

Assume the opposite and take $x \in \bigcap_{0}^{k+1} \pi_{H}\left(A^{i}\right)$. Since $x \in \pi_{H}\left(A^{k+1}\right) \subset \pi_{H}\left(\Lambda^{+}\right)$, then $x \in \pi_{H}\left(A^{i} \cap \Lambda^{+}\right)$, for $i=0, \ldots, k$, which is a contradiction because $\bigcap_{0}^{k} \pi_{H}\left(A^{i} \cap\right.$ $\left.\Lambda^{+}\right) \neq \varnothing$ implies $\bigcap_{0}^{k} \pi_{H_{0}}\left(\pi_{H}\left(A^{i} \cap \Lambda^{+}\right)\right)=\bigcap_{0}^{k} \pi_{H_{0}}\left(A^{i} \cap \Lambda^{+}\right) \neq \emptyset$.

Our first result characterizes families of convex sets with the $(k+1)$-intersection property.

Theorem 3.2. Let $F=\left\{A^{1}, \ldots, A^{d}\right\}$ be a family of $d$ convex sets in $R^{n+k}, d \geq k+1$. Every subfamily of $F$ with $k+1$ members has a common point if and only if

$$
\mu\left(T_{n}(F)\right) \geq k
$$

Proof. Suppose every subfamily of $F$ with $k+1$ members has a common point. We start by constructing a continuous map $\psi: G(n+k, n) \rightarrow T_{n}(F)$ as follows: for every $n$-plane $H$ through the origin, let $\pi_{H}: R^{n+k} \rightarrow H^{\perp}$ be the orthogonal projection, where $H^{\perp}$ is the $k$-plane through the origin orthogonal to $H$. We consider the family $\pi_{H}(F)=$ $\left\{\pi_{H}\left(A^{1}\right), \ldots, \pi_{H}\left(A^{d}\right)\right\}$ of $d$ convex sets in $H^{\perp}$. Note that every subfamily of $\pi_{H}(F)$ with $k+1$ members has a common point. Therefore, by Helly's theorem, the convex set $F(H)=\bigcap_{1}^{d} \pi_{H}\left(A^{i}\right)$ is not empty. Note also that $F(H) \subset H^{\perp}$ depends continuously on $H \in G(n+k, n)$. Let $\psi(H)$ be the $n$-plane through the center of mass of $F(H)$ and orthogonal to $H^{\perp}$. By construction, $\psi(H) \in T_{n}(F)$.

Let $i: T_{n}(F) \rightarrow G(n+k+1, n+1)$ and note that $i \psi: G(n+k, n) \rightarrow G(n+k+1, n+1)$ is homotopic to the inclusion. Therefore, by (2.1) and $(2.3),[0, k, \ldots, k]$ is not zero in $T_{n}(F)$ and hence $\mu\left(T_{n}(F)\right) \geq k$.

Suppose now $\mu\left(T_{n}(F)\right) \geq k$ and suppose that $\bigcap_{1}^{k+1} A^{i}=\emptyset$. By Lemma 3.1, there is a $k$-dimensional linear subspace $H$ of $R^{n+k}$ with the property that $\bigcap_{1}^{k+1} \pi_{H}\left(A^{i}\right)=\emptyset$, where $\pi_{H}: R^{n+k} \rightarrow H$ is the orthogonal projection. This is a contradiction because, by Lemma 2.1 , there is $\Gamma \in T_{n}(F)$ such that $\pi_{H}(\Gamma)$ is a single point which lies in $\bigcap_{1}^{d} \pi_{H}\left(A^{i}\right)$. This completes the proof of Theorem 3.2.

Example 3.1. For $k=1$ and $n=2$, Theorem 3.2 states that every two members of $F$ have a common point if and only if for every direction there is a transversal plane to $F$ orthogonal to it.

Our next result characterizes families of $k+2$ convex sets with the $(k+1)$-intersection property. Note that this time our transversals need not be of dimension $k$. 
Theorem 3.3. Let $F=\left\{A^{1}, \ldots, A^{k+2}\right\}$ be a family of $k+2$ convex sets in $R^{n+k}$ and consider an integer $1 \leq m \leq n$. Every subfamily of $F$ with $k+1$ members has a common point if and only if

$$
\mu\left(T_{m}(F)\right) \geq k .
$$

Proof. Suppose every subfamily of $F$ with $k+1$ members has a common point. For $i=1, \ldots, k+2$, let $a_{i} \in \bigcap_{j \neq i}\left\{A^{j} \in F\right\} \neq \emptyset$ and let $\Gamma$ be an $(m+k)$-plane containing $\Theta=\left\{a_{1}, \ldots, a_{k+2}\right\}$. Furthermore, for $i=1, \ldots, k+2$, let $B^{i} \subset \Gamma$ be the convex hull of the set $\left\{a_{j} \in \Theta \mid i \neq j\right\}$. Therefore, $F^{\prime}=\left\{B^{1}, \ldots, B^{k+2}\right\}$ is a family of convex sets in the $(m+k)$-plane $\Gamma$ with the property that $T_{m}\left(F^{\prime}\right) \subset T_{m}(F)$ because, for $i=1, \ldots, k+2$, $B^{i} \subset A^{i}$. By Theorem 3.2, for $n=m, \mu\left(T_{m}\left(F^{\prime}\right)\right) \geq k$, which immediately implies that $\mu\left(T_{m}(F)\right) \geq k$.

Suppose now $\mu\left(T_{m}(F)\right) \geq k$ and suppose $\bigcap_{1}^{k+1} A^{i}=\emptyset$. By Lemma 3.1, there is a $k$ dimensional linear subspace $H$ of $R^{n+k}$ with the property that $\bigcap_{1}^{k+1} \pi_{H}\left(A^{i}\right)=\emptyset$, where $\pi_{H}: R^{n+k} \rightarrow H$ is the orthogonal projection. Note now that $T_{m}(F) \subset M(m+(n-m+$ $k), m)$ is a collection of $m$-planes in $R^{m+(n-m+k)}$ with the property that $\mu\left(T_{m}(F)\right) \geq k$, and $H$ is a $k$-plane, $1 \leq k \leq n-m+k$. By Lemma 2.1, there is $\Gamma \in T_{m}(F)$ such that $\pi_{H}(\Gamma)$ is a single point which lies in $\bigcap_{1}^{k+1} \pi_{H}\left(A^{i}\right)$. This is a contradiction.

Example 3.2. For $k=1$ and $m=1$, Theorem 3.3 states that three convex sets have the property that every two of them have a common point if and only if there is a cycle of transversal lines to them.

We conclude with our main result, whose proof follows immediately from Theorems 3.2 and 3.3 .

Theorem 3.4. Let $F=\left\{A^{1}, \ldots, A^{d}\right\}$ be a family of $d$ convex sets in $R^{n+k}, d \geq k+2$, and consider an integer $1 \leq m \leq n$. Every subfamily $F^{\prime}$ of $F$ with $k+2$ members has the property that $\mu\left(T_{m}\left(F^{\prime}\right)\right) \geq k$ if and only if $\mu\left(T_{n}(F)\right) \geq k$.

Example 3.3. Following Horn and Klee's spirit, for $k=1, n=2$ and $m=1$, Theorem 3.4 states that every three convex sets of $F$ have a cycle of transversal lines if and only if $F$ has transversal planes orthogonal to every direction.

Example 3.4. For $m=n$, Theorem 3.4 states that if for every subfamily $F^{\prime}$ of $F$ with $k+2$ members and for every linear $n$-subspace of $R^{n+k}$ there is a translate which is an $n$-transversal to $F^{\prime}$, then every linear $n$-subspace of $R^{n+k}$ admits a translate which is an $n$-transversal to $F$.

Example 3.5. Let $F=\left\{A^{1}, \ldots, A^{d}\right\}$ be a family of convex sets in $R^{n+k}$. According to [1], $F$ has a virtual $n$-point if there are (homologically) as many $n$-transversals to $F$ as if $F$ had a common point, that is, as many $n$-transversals as there are $n$-planes through the origin in $R^{n+k}$. More precisely, $F$ has a virtual n-point if and only if $\mu\left(T_{n}(F)\right) \geq k$. For $m=n$, Theorem 3.4 states that every subfamily $F^{\prime}$ of $F$ with $k+2$ members has a virtual $n$-point if and only if $F$ has a virtual $n$-point. 


\section{References}

1. J. Arocha, J. Bracho, L. Montejano, D. Oliveros and R. Strausz, Separoids, their categories, and a Hadwigertype theorem for transversals, Discrete Comput. Geom., this issue, pp. 377-385.

2. J. Bracho, L. Montejano and D. Oliveros, The topology of the space of transversals through the space of configurations, Topology Appl., to appear.

3. S. S. Chern, On the multiplication in the characteristic ring of a sphere bundle, Ann. of Math. 49 (1948), 362-372.

4. L. Danzer, B. Grünbaum, and V. Klee, Helly's theorem and its relatives, in Convexity, Proceedings of Symposia in Pure Mathematics, Vol. 2, American Mathematical Society, Providence, RI, 1963, pp. 101180.

5. A. Horn, Some generalizations of Helly's theorem on convex sets, Bull. Amer. Math. Soc. 55 (1949), 923-929.

6. V. Klee, On certain intersection properties of convex sets, Canad. J. Math. 3 (1951), 272-275.

7. J. W. Milnor and J. D. Stasheff, Characteristic Classes, Annals of Mathematical Studies, No. 76, Princeton University Press, Princeton, NJ, 1974.

8. L. Montejano, Recognizing sets by means of some of their sections, Manuscripta Math. 76 (1992), 227-239.

9. L. S. Pontryagin, Characteristic cycles on differential manifolds, Trans. Amer. Math. Soc. 32 (1950), 149218.

Received October 25, 2000, and in revised form September 27, 2001, and October 17, 2001.

Online publication March 1, 2002. 